\title{
Quantifying the Impacts of Acoustic Target Detections Using a Range-Independent Model Versus Range-Dependent Model
}

\author{
Talia Beech \\ Defence Research and Development Canada \\ Centre for Operational Research and Analysis \\ Ottawa, Ontario, Canada \\ talia.beech@forces.gc.ca \\ Ernest Jankowski \\ Naval Engineering Test Establishment \\ Halifax, NS, Canada \\ ernest.jankowski@forces.gc.ca \\ Received 3 November 2017 \\ Revised 26 April 2018 \\ Published 14 June 2018
}

\begin{abstract}
The purpose of this study is to identify and quantify the difference in detections of a subsurface target from a subsurface sensor source between range-independent and range-dependent versions of the same acoustic propagation model. Environmental data were pulled from open source databases to provide an application for the comparison and using novel measures of merit, the authors were able to quantify the difference in detection performance between models. This study suggests that provided multiple types of environments are considered, it is possible to use a range-independent model to give good approximations to the accuracy of detections, one would achieve using a full range-dependent sound propagation model.
\end{abstract}

Keywords: Range independent; range-dependent; acoustic modeling; sensor; target; environment; Scotian Shelf; detection.

\section{Introduction}

When choosing the types of models to use for an acoustic study, one can consider two fundamental types of sound propagation model: a range-independent or range-dependent one. This question must be considered carefully, since the requirements of processing and

This is an Open Access article published by World Scientific Publishing Company. It is distributed under the terms of the Creative Commons Attribution 4.0 (CC-BY) License. Further distribution of this work is permitted, provided the original work is properly cited. 


\section{T. Beech E E. Jankowski}

the amount of data can be significantly higher for the range-dependent case. There are several types of acoustic propagation models available for use. To name a few: ray tracing, normal mode, multi-path expansion, parabolic equation, etc., all of which are described by Jensen! 1 We chose to use the Bellhop model, 2 in both range-dependent and rangeindependent versions to carry out this study, primarily due to the ease of availability and popularity of use amongst acousticians. The range-independent version of Bellhop model uses a fixed bottom depth, a single, static, range-independent sound speed profile (SSP) and a single, static, range-independent bottom reflection loss submodel or table. Comparatively, in reality, SSPs, bathymetry and reflection loss are all location dependent and vary with range and azimuth within a single scenario. This reality is what is driving the requirement for this study, to better understand the impact of using a range-independent model to approximate what we know is real: a range-dependent environment in which sound travels. In the sections that follow range-independent and range-dependent versions of the Bellhop model will be compared to investigate the effects of using a range-independent underwater acoustic propagation model in an area known as the Scotian Shelf, where there exists a case of pronounced range dependence across the continental shelf break.

\subsection{Range-dependent propagation model}

The range-dependent underwater sound propagation model called DRDC Bellhop ${ }^{3}$ and Ref. 4 is a derivative of an open source Bellhop model developed by Mike Porter ${ }^{5-17}$ and available from the Oceans Acoustic Library. 8 The model accepts range-dependent inputs for bathymetry, SSPs and bottom reflection loss. Inputs for the model are discussed in detail in Refs. 3 and 4. The surface loss model, the Beckmann-Spizzichino Surface Loss Model, was used with the wind speed of 15 knots; $1.6 \mathrm{kHz}$ frequency ${ }^{910}$ The model returns a matrix with transmission loss (TL) values for a predefined grid of receiver ranges and depths that the user defines.

The signal excess (SE) for a passive sonar is given by the sonar equation as defined by Urick ${ }^{11}$ For a given passive sonar (with a fixed processing mode and fixed acoustic source strength), the final SE will depend only on the TL:

$$
\mathrm{SE}=\mathrm{TL}_{*}-\mathrm{TL} .
$$

By definition, "SE $=0$ " corresponds to the probability of detection of $50 \%$ at a selected value of the probability of false alarm. The value of TL that corresponds to the "SE $=0$ " is denoted by $\mathrm{TL}_{*}$ (we assume that the ambient noise is fixed and location independent and that the source strength does not change throughout the scenario duration). The TL is further translated into the probability of detection value using a transition curve from Ref. 11, which is representative for a number of sonars tested at sea (Fig. 12.10 from Ref. 11, p. 388, compares at sea detections with the theoretical detection curve). SE $=0$ and the corresponding probability of detection of $50 \%$ is shown in the transition curve is plotted in the panel on the left in Fig. 1]. 

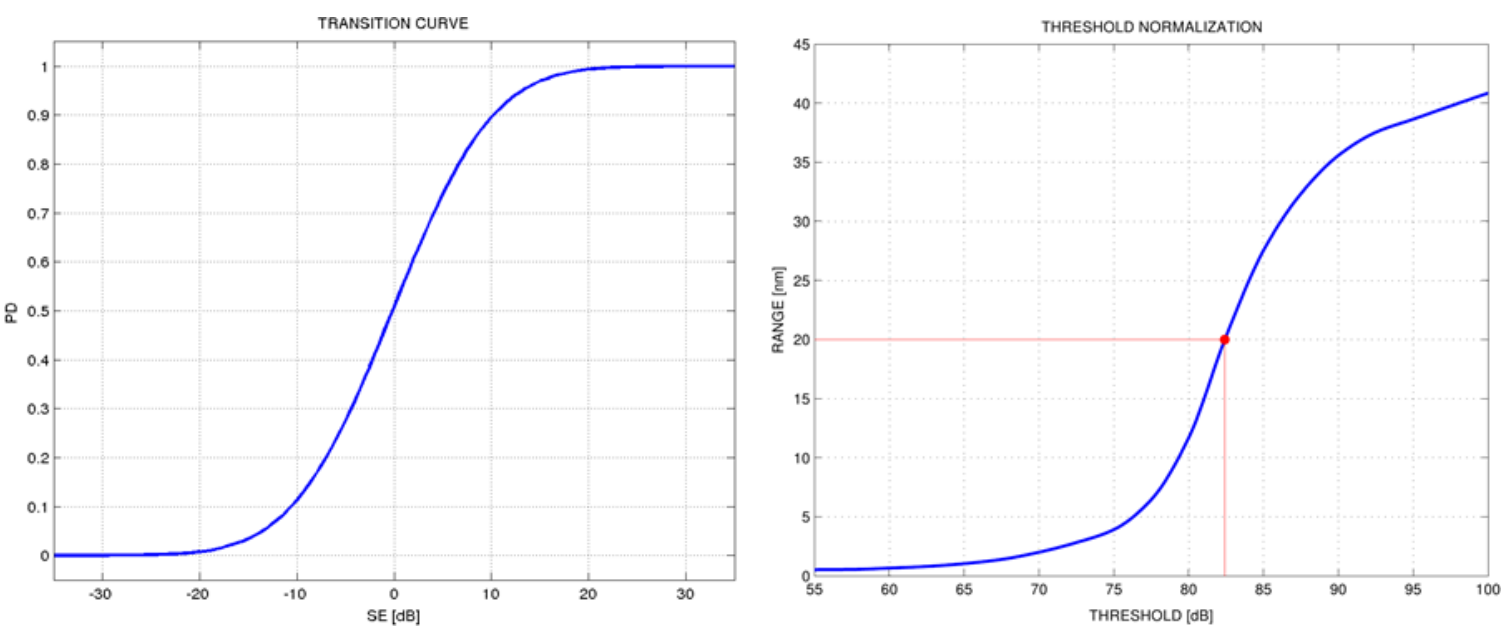

Fig. 1. Transition curves: the panel on the left shows the probability of detection as a function of the SE (based on Ref. 11) and the panel on the right shows the average detection range as a function of $\mathrm{TL}_{*}$ threshold for a subsample of 1000 combinations of latitude, longitude and source depth points sampled from the locations, azimuths, source depths and receiver depths superimposed.

The detection range (for a given sonar receiver depth, $z$ ), $r_{\operatorname{det}}(z)$, is defined as the maximum range (at depth $z$ ) for which the probability of detection reaches $50 \%$. That is,

$$
r_{\text {det }}(z)=\max \left(\left\{r: p_{\operatorname{det}}(z, r) \geq 0.5\right\}\right) .
$$

The value of $\mathrm{TL}_{*}$ is sonar-specific and also depends on the source strength, receiver capabilities (self-noise, beamforming) and processing details such as bandwidth and integration period as well as the level of ambient noise. $\mathrm{TL}_{*}$ is chosen somewhat arbitrarily, however, it is realistic to the types of ranges one may want to achieve reliable detections.

In this study, the authors assume a mean detection range of $20 \mathrm{~nm}$. This is a plausible detection range, specifically, if one considers ranges in terms of making detections of acoustic sources like marine mammals or in searching for items such as debris, shipwrecks, etc. The $20 \mathrm{~nm}$ notional detection range corresponds to the average detection range over all considered combinations of latitude, longitude, source and receiver depths. It includes detections in convergence zones as well as long as the sound intensity is sufficient. The individual detections will be statistically distributed (with a mean value of $20 \mathrm{~nm}$ ). More specifically, we normalize the source level, ambient noise level and all sonar specific parameters in such a way that the mean detection range amounts to $20 \mathrm{~nm}$. The relationship between mean detection range and $\mathrm{TL}_{*}$ is described as the curve shown in the panel on the right in Fig. $\mathbb{1}$. In creating this figure, the mean detection range was evaluated over a subsample of 1000 latitude, longitude and source depth combinations. The data set is described in detail in the succeeding section.

The authors further focus on the distribution of detection ranges and differences between range-independent and range-dependent computations. The authors also wish to identify 
the importance of the realization that all subsurface detections are strongly dependent not only on the environment, but also are sonar- and target-specific.

\subsection{Range independent model}

The DRDC Bellhop propagation model is also used for range-independent computations. However, the model is used with one simplification to the range-dependent case: the central (at range zero) SSP, depth and reflection loss inputs are used through the entire range profile. This is described in the following data sample discussion. The corresponding detection range and probability of detection obtained from the range-independent computations are labeled with index zero: $r_{\operatorname{det} 0}$ and $p_{\text {det0 }}$, respectively.

\section{Data Sources}

The authors were able to obtain bathymetry, ${ }^{[12]}$ sea temperature and salinity ${ }^{13}$ data from the Effects of Sound on Marine Environment (ESME) research project by the Boston University Hearing Research Center and the US Office of Naval Research. The data are openly available from https://esme.bu.edu. The sea temperature and salinity depth profiles are used to compute the SSPs using the Mackenzie formula, which is described in detail in Ref. 14. Monthly averages for the sea temperature and salinity (as functions of depth) were used for the randomly chosen month of September. Any temporal (day-to-day) variability or uncertainties associated with the water temperature and salinity profiles that will affect the sound velocity were insignificant and not considered. The authors used the DRDC Bellhop option that utilizes the US Navy High Frequency Bottom Loss (HFBL) model applicable to frequencies in the range of $1.5-4.0 \mathrm{kHz} .15$ The model assigns the bottom loss based on the value of grazing angle and the Maritime Geophysical Survey (MGS) province number (an integer between 1 and 9). The HFBL model is based on the in-field reflection loss measurements obtained by the MGS. The map of MGS province numbers for the area of interest was taken from Ref. 16. Based on the information provided in Ref. 15, MGS province number of two has been used for the shallow area of Scotian Shelf and number nine for the area of the (rapid) transition from shallow to deep water.

\subsection{Data specifications}

The latitude-longitude locations of data points are uniformly sampled from a box defined by $\left(41^{\circ} \mathrm{N}, 65^{\circ} \mathrm{W}\right)$ and $\left(45^{\circ} \mathrm{N}, 62^{\circ} \mathrm{W}\right)$. Figure 2 shows the latitude-longitude locations where data points were sampled against the bathymetry and MGS province numbers map. For each latitude-longitude location, multiple source depths are considered from $5 \mathrm{~m}$ to maximum depth or $600 \mathrm{~m}$ (whichever is smaller) with a step of $10 \mathrm{~m}$. For each latitude, longitude and source depth combination, 36 azimuthal cuts are considered (every $10^{\circ}$ starting from the North). Each azimuthal cut extends $100 \mathrm{~km}$ in range. Figure 2 also illustrates the azimuthal cuts, for an example, location (the cuts are plotted against the bathymetry or MGS province number backgrounds) shown by the white lines through a sample data point. 

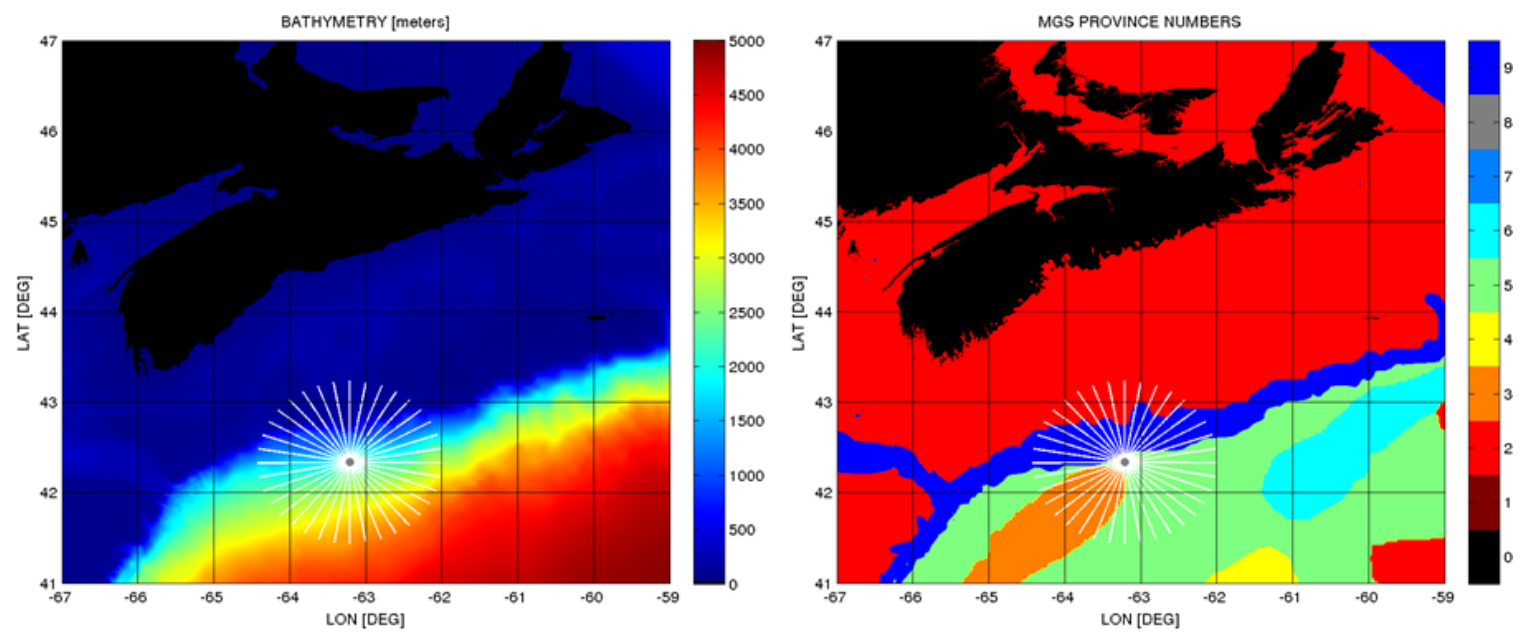

Fig. 2. Location of all dataset points plotted against bathymetry and MGS province number background map. All 36 azimuthal cuts for an example location $\left(42.3373^{\circ} \mathrm{N}, 63.2101^{\circ} \mathrm{W}\right.$ ) against (a) bathymetry and (b) MGS province number background map.

A combination of latitude, longitude, source depth and azimuth cuts constitutes a single DRDC Bellhop run, which we often refer to as the "Bellhop cut". Some examples of the Bellhop cuts are shown in Fig. 3, for the location identified in Fig. 2. using the azimuthal cuts at $40^{\circ}$ intervals, from $0^{\circ}$ through to $360^{\circ}$, and the last panel in Fig. 3 representing the rangeindependent case. In addition, for each latitude, longitude and source depth combination, a uniform reference profile is created by using the central values for the depth, MGS province and SSP. This additional cut represents the range-independent environmental conditions. This reference cut is labeled as azimuth " $\mathrm{AZ}=800$ " in Fig. 3

The data for receiver depth are gathered at an interval every $10 \mathrm{~m}$ from 0 to $600 \mathrm{~m}$ or the bottom depth in areas shallower than $600 \mathrm{~m}$. Cumulatively, this equals 60 detection ranges $r_{\text {det }}(z)$ for a single cut and over 60 million detection ranges in total. These totals are further broken down in Table 1 .

\section{Measures of Merit}

Three measures can be used to quantify the discrepancy between sonar performance results obtained in a range-independent and range-dependent models:

- the error between the two models in detection range;

- the mean difference between the two models in probability of detection and

- the relative difference in detection volume.

These three measures are defined in this section.

The error in the detection range is defined as the difference between the rangeindependent approximation $\left(r_{\operatorname{det} 0}\right)$ and the range-varying environment result $\left(r_{\text {det }}\right)$ :

$$
\Delta r_{\operatorname{det}}(z)=r_{\operatorname{det} 0}(z)-r_{\operatorname{det}}(z) .
$$



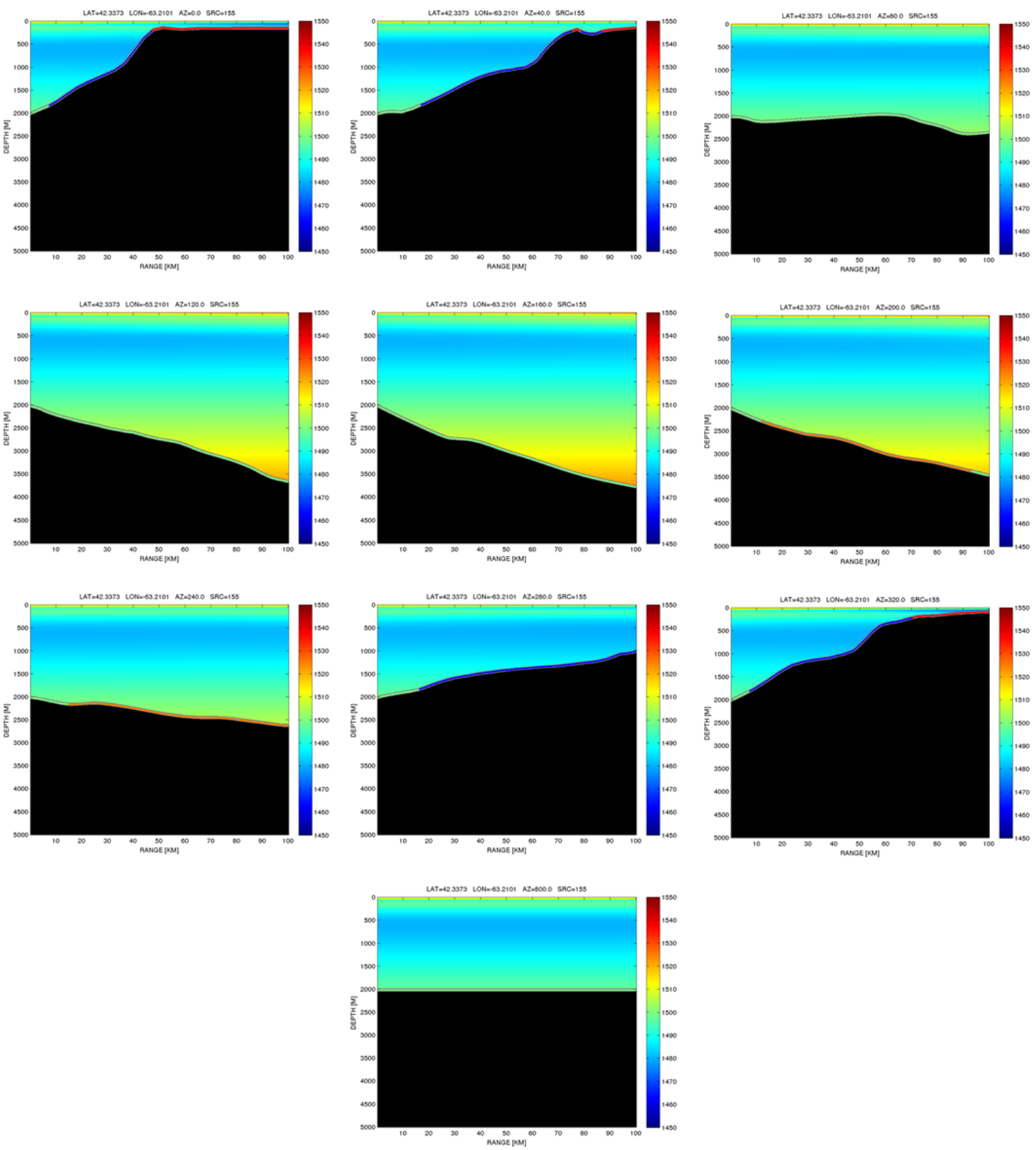

Fig. 3. Selected Bellhop input cuts for the location from Fig. 2 $42.3373^{\circ} \mathrm{N}, 63.2101^{\circ} \mathrm{W}$, azimuths (left to right, top to bottom $)=0^{\circ}, 40^{\circ}, 80^{\circ}, \ldots, 320^{\circ}$. The last panel corresponds to the range-independent environment cut obtained from extending uniformly the environment properties at $42.3373^{\circ} \mathrm{N}, 63.2101^{\circ} \mathrm{W}$ (range 0 ) out to the maximum range of $100 \mathrm{~km}$. The bottom line color indicates the MGS province using the color coding defined in Fig. 2. 
Table 1. Description of the dataset used in the study.

\begin{tabular}{ll}
\hline Total Number of Points & \multicolumn{1}{c}{ Data Type } \\
\hline 884 & Latitude-longitude locations \\
32032 & Latitude, longitude and source depth combinations \\
1153152 & Azimuthal cuts (excluding range independent) \\
1185184 & Azimuthal cuts (including range independent) \\
60062064 & Considered ranges (range error values) \\
\hline
\end{tabular}

A positive value of $\Delta r_{\text {det }}$ means that the range-independent approximation overestimates the detection range, and conversely, a negative value means that the range-independent approximation underestimates the detection range. $\Delta r_{\text {det }}$ is always computed at the same receiver depth $z$.

The mean difference in detection probability is defined as

$$
\Delta P_{\text {det }}=\frac{\int_{V_{\mathrm{c}}}\left|p_{\operatorname{det} 0}-p_{\text {det }}\right| d V}{\int_{V_{\mathrm{c}}} d V} .
$$

The integration volume $V_{c}$ is the common volume for range-independent and rangedependent environments extending from sea surface down to the maximum depth evaluated. The common volume is defined as the volume not occupied by land in any of the either range-independent or range-dependent cases. The measure $\Delta P_{\text {det }}$ can be evaluated for each separate azimuthal cut (commonly referred to as bearing line in an operational context) or collection of azimuthal cuts for each data point.

The relative difference in the detection volume is defined as

$$
\Delta V_{\mathrm{det}}=\frac{\int_{V_{\mathrm{c}}} p_{\mathrm{det} 0} d V-\int_{V_{\mathrm{c}}} p_{\operatorname{det}} d V}{\int_{V_{\mathrm{c}}} p_{\operatorname{det}} d V} .
$$

This detection volume is interpreted as volume weighted by the probability of detection. Similarly, as $\Delta P_{\text {det }}, \Delta V_{\text {det }}$ can be computed for each azimuthal cut separately or for all azimuthal cuts for each data point.

\section{Results}

Example results are shown for the single data point, i.e. a single combination of latitude, longitude and source depth: $42.3373^{\circ} \mathrm{N}, 63.2101^{\circ} \mathrm{W}, 155 \mathrm{~m}$, as described in Fig. 2 Results in Fig. 4 show the detection ranges superimposed for all azimuthal cuts: $0^{\circ}$ to $360^{\circ}$ with $10^{\circ}$ steps across the $100 \mathrm{~km}$ range. The red circles correspond to the range-independent environment. There is one such point for each receiver depth (the vertical axis shows receiver depth and the horizontal axis shows range). The blue circles show the detection ranges for 


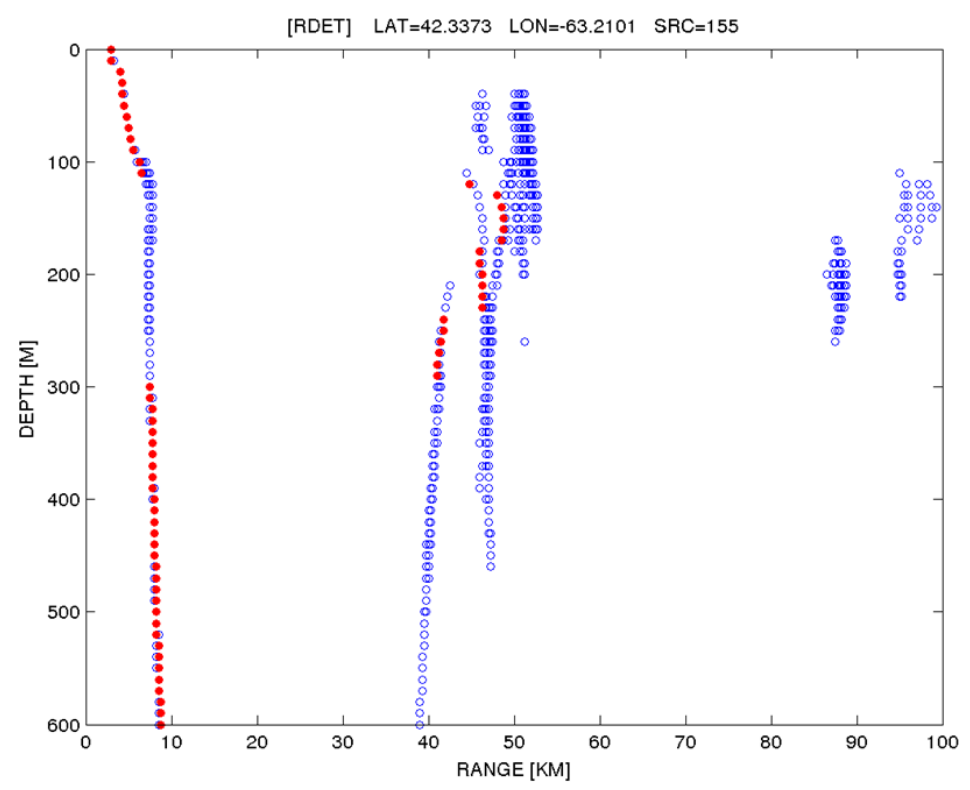

Fig. 4. Detection ranges for $42.3373^{\circ} \mathrm{N}, 63.2101^{\circ} \mathrm{W}, 155 \mathrm{~m}$ (source depth) as a function of receiver depth, over all azimuths.

each azimuthal cut. It can be noted that the detection ranges can vary significantly between each azimuthal look (from the range-independent environment) as some detections occur in the convergence zones that show only for certain azimuths.

These results can be better understood by considering the TL, probability of detection $\left(p_{\text {det }}\right)$ and probability of detection difference $\left(p_{\text {det } 0}-p_{\text {det }}\right)$ between the range-independent and range-varying environments. It can be inferred when considering the results that all down-sloping (southward) profiles show a strong convergence zone that is not present in the up-sloping (northward) profiles. The range-independent profile features a moderate convergence zone: the range-independent environment underestimates the probability of detection in significant water volume for the southward profiles and overestimates it for the northward profiles. Similar opening and closing of a convergence zone is a common feature for locations on the transition between deep and shallow water.

The close-range detections (detections that are less than $10 \mathrm{~km}$ from the receiver, shown in Fig. (4) correspond to primary detections, the mid-range detections correspond to the first convergence zone and the far-end detections correspond to the second convergence zone. For this particular example, close to the source, the sound refracts towards the bottom very strongly and at a given fixed depth, the sound intensity decreases very fast with range making the detection beyond approximately $10 \mathrm{~km}$ impossible. For some azimuthal cuts, the water depth may be sufficiently large that the sound will eventually start to refract toward the surface leading to the first convergence zone: the mid-range detections may occur. If the water depth is not sufficiently large (for some azimuthal cuts), the sound will intersect with the bottom before the upward refraction could start. (The reflected intensity may typically be insufficient for the detection.) Similar pattern may occur for the second convergence zone. 

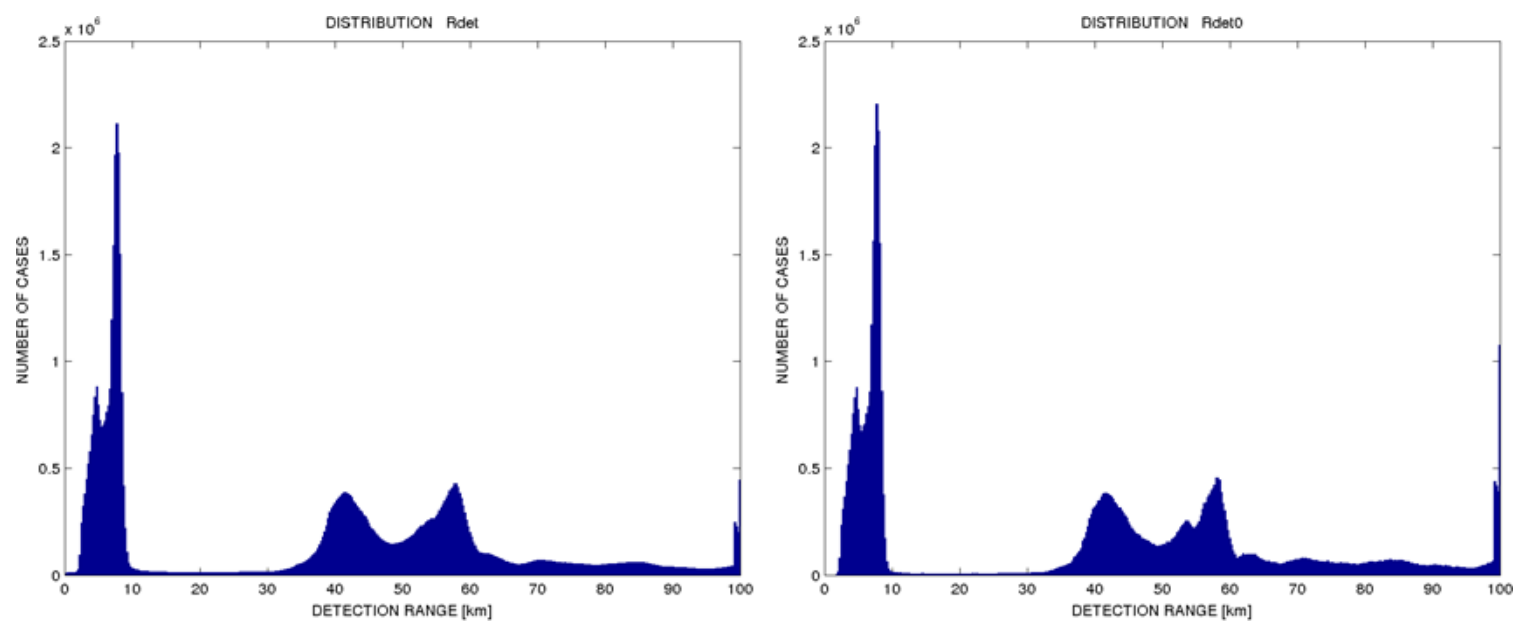

Fig. 5. Distribution of detection ranges for the range-dependent case in the panel on the left and the rangeindependent case in the panel on the right.

\section{Detection Range Statistics}

The distribution of over 60 million of detection ranges for the entire dataset is shown in Fig. 5. 32032 combinations of latitude, longitude and source depths times 36 azimuthal cuts for each combination and times up to 60 receiver depths per each cut (see Table 1). It is not surprising that a very large spread in the detection ranges is observed as the dataset covers a large variety of water conditions with some cases featuring multiple convergence zones. Figure 5 shows some very interesting results: the distributions of $r_{\text {det }}$ for both cases are very similar, that is the range-independent case is showing very similar detection ranges to the range-dependent case.

The histogram in the left panel of Fig. 6] shows the distribution of $\Delta r_{\text {det }}=r_{\text {det0 }}-r_{\text {det }}$ over an entire data sample. The distribution has a very tall peak around zero and very broad tails. For majority of the detection ranges, the error resulting from the range independent case is within $5 \mathrm{~km}$ bounds. The histogram in the right panel of Fig. 6 is a zoomed in version of the histogram in the left panel with the central part (the tall peak) removed in order to expose the tails and delve deeper into the distribution of the data on the far left and right sides of the histogram. It can be noted that the range errors can be significant and also that there are more points on the positive side of $\Delta r_{\text {det }}$, i.e. the range-independent approximation more often overestimates the detection ranges than underestimates. This asymmetry in the distribution results, to some extent, from the fact that for some depths, the detection range is limited by hitting the bottom. However, this is not the only reason for the asymmetry in results. It is observed that in some cases, the probability of detection is decreased past the bottom of a trough even at receiver depths for which the detection is obstructed by land. This can be further examined by looking at the cumulative probability density function of the absolute value of the error. Table 2 summarizes the distribution of errors and Fig. 7 shows the cumulative probability of the absolute value of the error, 

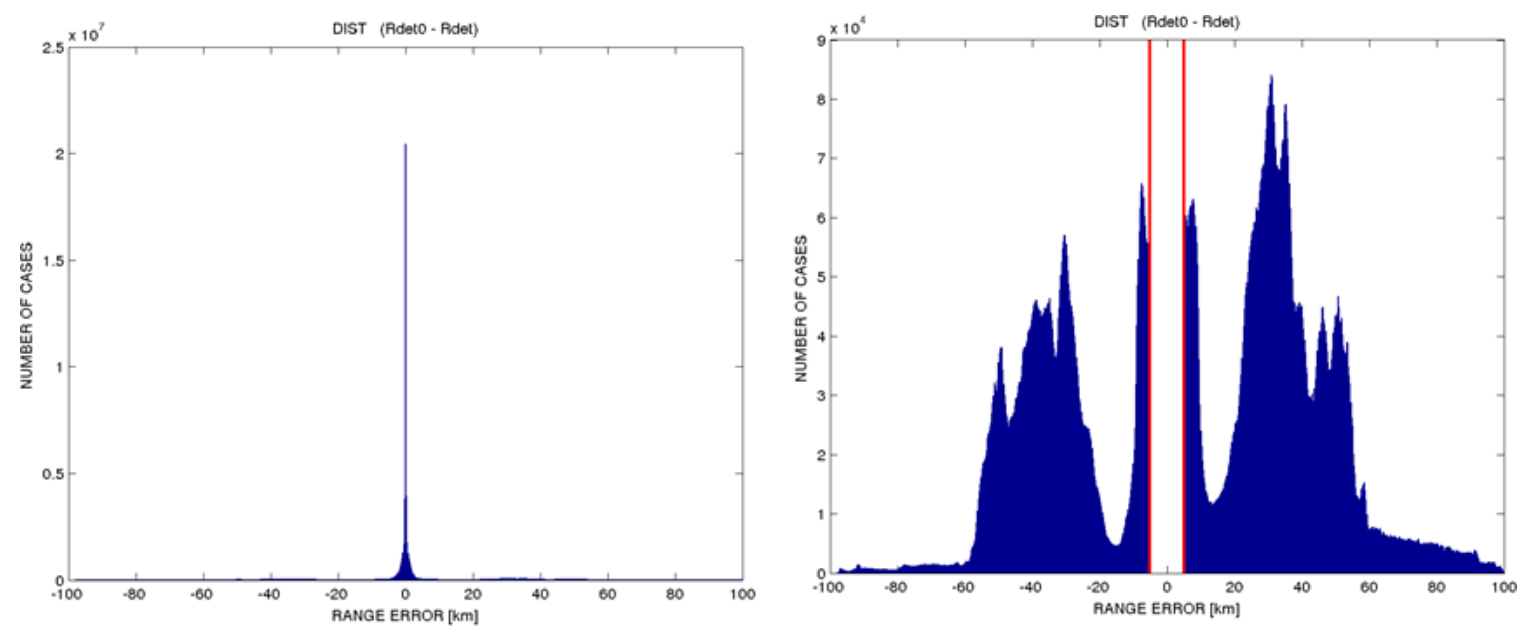

Fig. 6. (Color online) Distribution of range error, $\Delta r_{\text {det }}=r_{\text {det0 }}-r_{\text {det }}$ for the entire data set in the left panel, and for the smaller range interval $-5<\Delta r_{\text {det }}<5 \mathrm{~km}$ (with the middle interval removed, as indicated by the two red vertical lines) on the right. All latitude and longitude locations, azimuths, source and receiver depths superimposed.

Table 2. Percentages of data points with the detection range error $\left(\Delta r_{\text {det }}\right)$ within given bounds.

\begin{tabular}{rllcc}
\hline \multicolumn{3}{c}{ Range Error Limits } & $\%$ of Points \\
\hline$-\infty$ & $<\Delta r_{\text {det }}<-50.0 \mathrm{~km}$ & 1.3 \\
$-50.0 \mathrm{~km} \leq \Delta r_{\text {det }}<-20.0 \mathrm{~km}$ & 7.2 \\
$-20.0 \mathrm{~km} \leq \Delta r_{\text {det }} \leq-10.0 \mathrm{~km}$ & 0.5 \\
$-10.0 \mathrm{~km}$ & $\leq \Delta r_{\text {det }}<$ & $-5.0 \mathrm{~km}$ & 1.7 \\
$-5.0 \mathrm{~km}$ & $\leq \Delta r_{\text {det }}<$ & $-1.0 \mathrm{~km}$ & 6.6 \\
$-1.0 \mathrm{~km} \leq \Delta r_{\text {det }} \leq$ & $1.0 \mathrm{~km}$ & 60.2 \\
$1.0 \mathrm{~km}$ & $\leq \Delta r_{\text {det }}<$ & $5.0 \mathrm{~km}$ & 6.4 \\
$5.0 \mathrm{~km} \leq \Delta r_{\text {det }} \leq$ & $10.0 \mathrm{~km}$ & 1.9 \\
$10.0 \mathrm{~km} \leq \Delta r_{\text {det }} \leq$ & $20.0 \mathrm{~km}$ & 1.0 \\
$20.0 \mathrm{~km}$ & $\leq \Delta r_{\text {det }}<$ & $50.0 \mathrm{~km}$ & 10.2 \\
$50.0 \mathrm{~km}$ & $\leq \Delta r_{\text {det }}<$ & $\infty$ & 2.9 \\
\hline
\end{tabular}

$\left|\Delta r_{\text {det }}\right|$, below the value on horizontal axis. This plot can be interpreted such that if the required accuracy is $1 \mathrm{~km}$, over $60 \%$ of the range computations are within this error, or if the required accuracy is $10 \mathrm{~km}$, then over $75 \%$ of the range computations are within this error. However, further inclusion of the data points (above $75 \%$ ) would have to lead to a very large bound on the absolute value of the range error.

From Fig. 6, it can be seen that there are many points for which $\Delta r_{\text {det }}=0$ (or $\approx 0$ ). Further investigation into these points show that most of these points are located around the range of approximately $8 \mathrm{~km}$. There is a class of points for which the SSP and bottom properties result in very short detection ranges. This in combination with the fact that the properties used for the range-independent profile correspond to those at the center of 


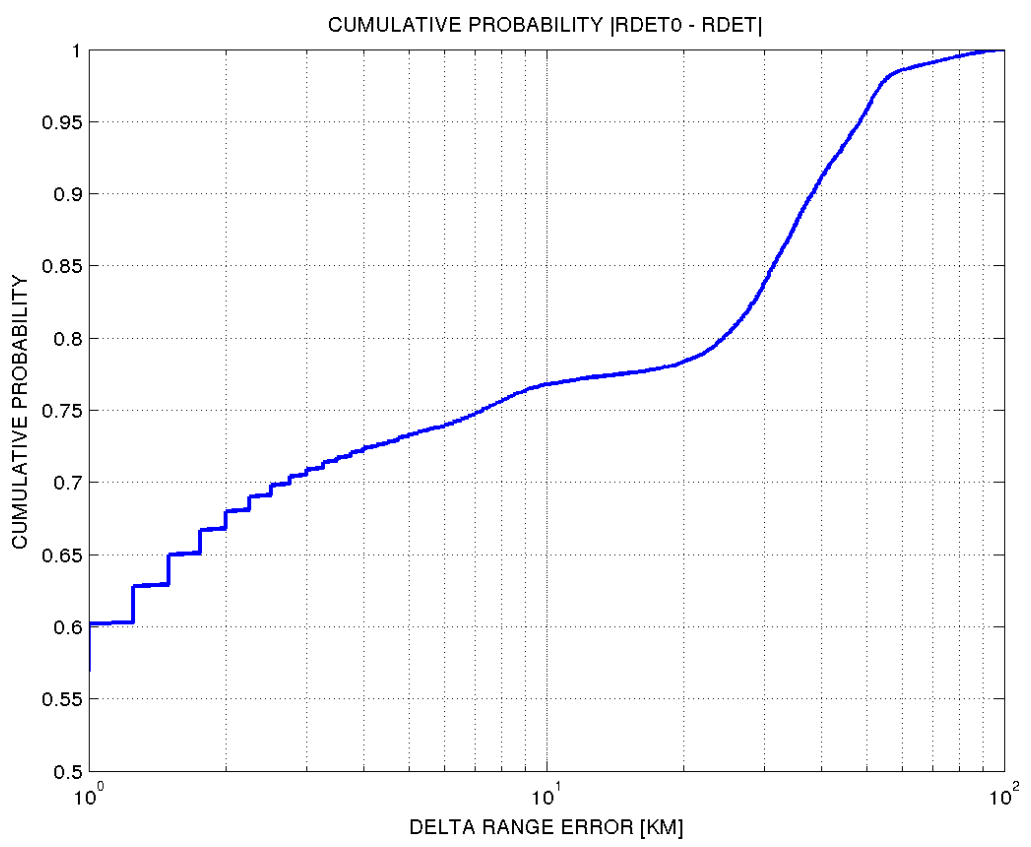

Fig. 7. The cumulative error distribution $\left|\Delta r_{\text {det }}\right|=\left|r_{\operatorname{det} 0}-r_{\text {det }}\right|$.

the range-varying profiles results in no significant difference in the sound TL and detection range.

Further statistics for $r_{\text {det }}, r_{\text {det0 }}$ and $\Delta r_{\text {det }}$ over the Scotian Shelf area are shown in Fig. 8. The top row in Fig. 8 compares the distributions of the range-independent case, $r_{\text {det0 }}$ (column one) and range-dependent cases, $r_{\text {det }}$ (column two) as functions of the ocean depth. The histograms are normalized in such a way that the sum of bins on each horizontal line is one. There are notable differences in detection ranges for shallow water between these two panels, most notably at depths up to $300 \mathrm{~m}$. At shallow, Scotian Shelf depths (the depth is defined as the ocean depth at the source location), the detection range values have much wider distribution in range-dependent case as compared to range-independent setup. In the former case, quickly changing bathymetry leads to more uniform distribution whereas in range-independent case, the results tend to be either very close range or very far detections if the ducting conditions arise. Also, both convergence zones are more pronounced in the range-independent model (panel top left). If the source is located in deep water, the conditions for convergence zones are more likely in range-independent case. In contrast, in range-dependent case, for some azimuths, the upslope bottom prevents the condition for convergence zone.

The panel in row two, column one shows correlation of $r_{\text {det }}$ error with the ocean depth: $\Delta r_{\text {det }}$ can be significant for shallow water and is most often positive (the range-independent model overestimates the detection ranges). This plot emphasizes that it is more likely to overestimate the detection range (than underestimate) when range-independent approximation is used. 

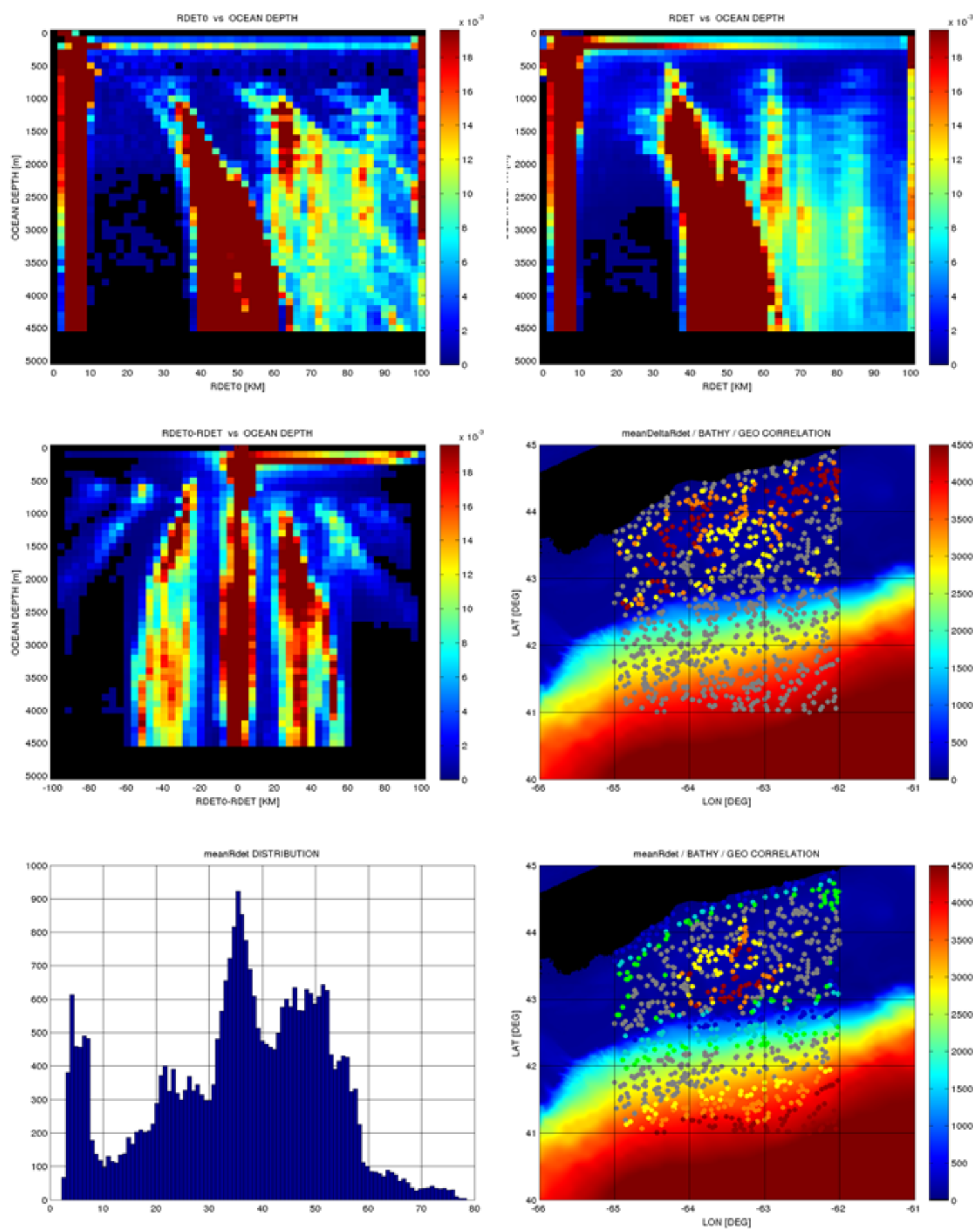

Fig. 8. Additional $r_{\text {det }}, r_{\text {det0 }}$ and $\Delta r_{\text {det }}$ statistics for range-independent and range-dependent models over ocean depth, bathymetry. See text for detailed description. 
Table 3. Color coding for locations sorted in increasing order of mean value of $r_{\text {det }}$.

\begin{tabular}{lc}
\hline First $10 \%$ of locations (with the smallest mean $r_{\text {det }}$ ) & Blue \\
$10-20 \%$ of locations & Cyan \\
$20-30 \%$ of locations & Green \\
$30-70 \%$ of locations & Gray \\
$70-80 \%$ of locations & Yellow \\
$80-90 \%$ of locations & Orange \\
last $10 \%(90-100 \%)$ & Red \\
\hline
\end{tabular}

The panel in row two, column two shows the locations of outliers for mean $\left|\Delta r_{\text {det }}\right|$. For each location (latitude, longitude pair), the mean of $\left|\Delta r_{\text {det }}\right|$ is computed over all applicable receiver depths, all azimuths and all source depths. The red dots are the $10 \%$ of the worst case points (largest mean $\left|\Delta r_{\text {det }}\right|$ ); orange and yellow indicate subsequent $10 \%$ of locations when sorted by value of mean $\left|\Delta r_{\text {det }}\right|$ in decreasing order. This plot shows that largest discrepancies between range-dependent and range-independent cases arise on the Scotian Shelf as opposed to the deep water.

The panel in row three, column one shows the mean $r_{\text {det }}$ distribution (mean over all azimuths, receiver and source depths). The panel in row three, column two shows the geo-spatial locations of the smallest and the largest mean detection ranges for the rangedependent case. The color coding is explained in Table 3 The points with the shortest mean $r_{\text {det }}$ are located near the shore line and in the transition zone. (In both cases, this is partially explained by the fact that detections are limited by banks.) The points with largest mean detection ranges appear in the deep part of the ocean and in shallow water near latitude $43^{\circ} \mathrm{N}$ and longitude of $63.5^{\circ} \mathrm{W}$.

\section{Probability of Detection}

The distribution of $\Delta p_{\text {det }}$ given by Eq. (4) is illustrated in Fig. 9 The histogram in the top left panel has one entry for each combination of latitude, longitude and source depth; the histogram in the top right panel has one entry for each combination of latitude, longitude and source depth and azimuth. The two histograms illustrate that for majority of data points, the volume weighted probability of detection error is within $10 \%$ with modes around $5 \%$ and $0 \%$. These histograms also show that the distribution has long tails: cases with much higher value of the error are possible. The panel in the bottom row on the left shows locations of largest values of $\Delta p_{\text {det }}$ (red: worst 10\%, orange: next 10\%, yellow: next $10 \%$ and gray: all the rest). The panel on the bottom row on the right in Fig. 9 shows the correlation or $\Delta p_{\text {det }}$ with the ocean depth (depth taken at the center of the profile). The histogram is normalized so that the sum of bins on each horizontal line is one. It can be noted from both panels in the bottom row that the largest differences in $\Delta p_{\text {det }}$ are located in shallow water for the area studied. 

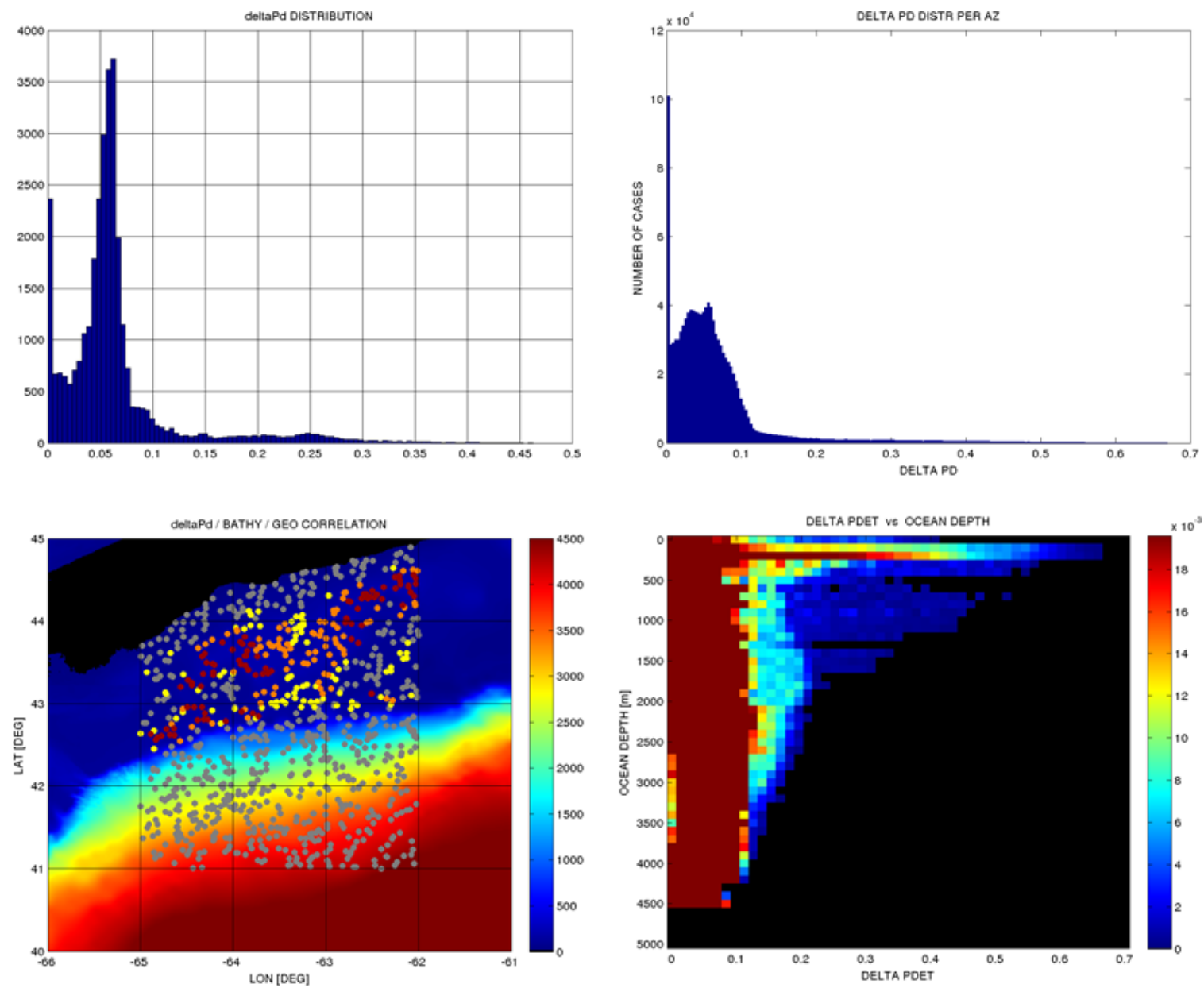

Fig. 9. Additional statistics for the difference in probability of detection between range-independent and range-dependent cases over ocean depth, bathymetry. See text for detailed description.

\section{Detection Volume}

Statistics are shown in Fig. 10 for the effective detection volume difference (defined by Eq. (5) ) between range-independent and range-dependent environments. The histogram in the top left panel of Fig. 10 contains entries for each combination of latitude, longitude and source depth considered in the study. The histogram in row two, column one has one entry for each azimuthal cut (36 entries for each combination of latitude, longitude and source depth considered in this study, which gives approximately 1.2 million points).

Histograms on the right in row one and two plot the same variables as the panels in the same rows on the left, respectively, but feature a zoomed in focus on the central parts (i.e. the scale has been adjusted in order to omit the large outliers).

Maps shown in the third row of Fig. 10 (data points plotted against bathymetry background) show geographic locations of outliers. For each point plotted in both panels in row 

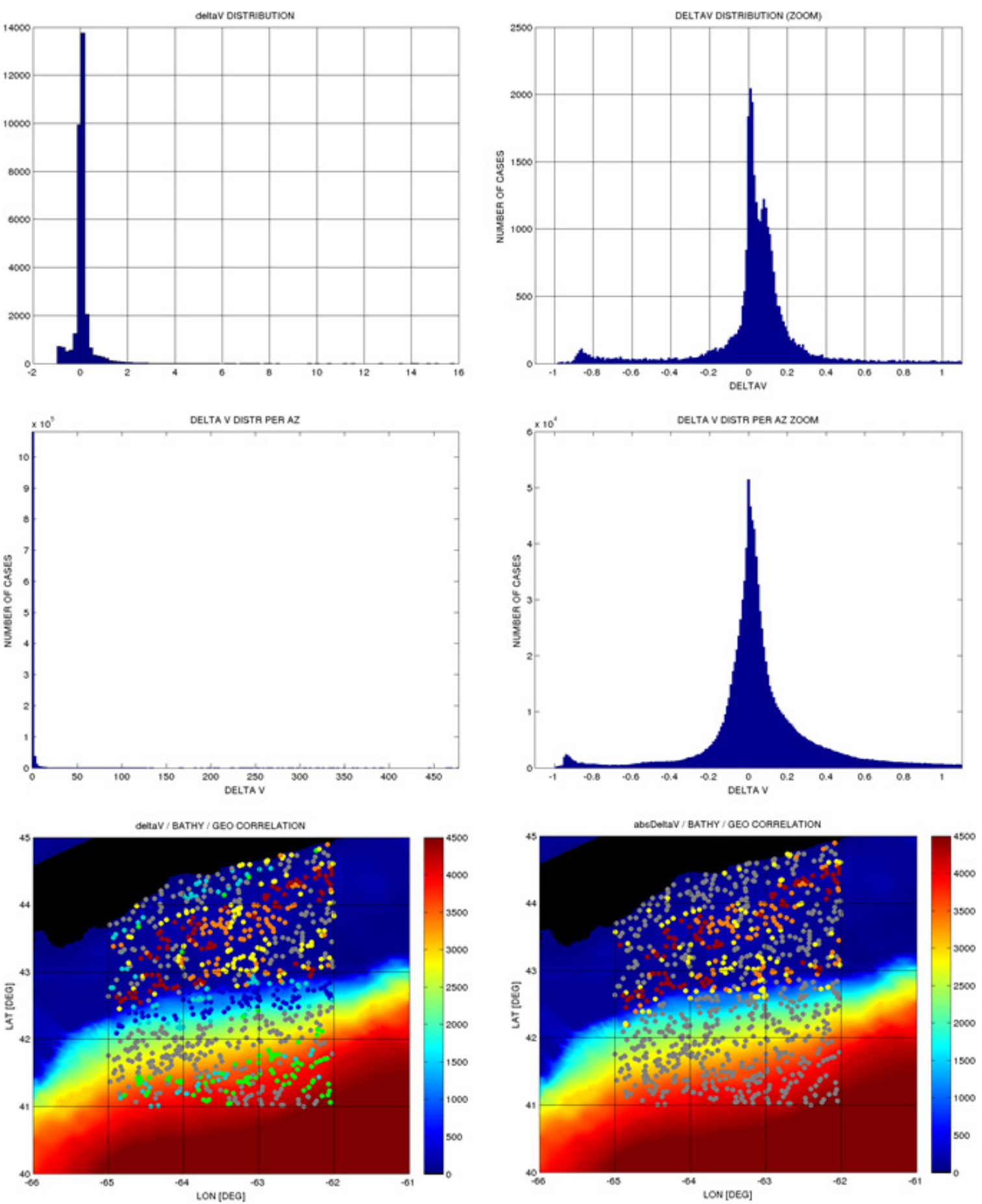

Fig. 10. Additional statistics for the difference in detection volume between range-independent and rangedependent cases over ocean depth, bathymetry. See text for detailed description. 
three, average over all source depths has been taken of $\Delta v_{\text {det }}$ or $\left|\Delta v_{\text {det }}\right|$ (absolute value of $\left.\Delta v_{\text {det }}\right)$, respectively. The color coding for the panel in row three on the left corresponds to that of Table 3. The color coding for the panel in row three on the right follows: red: worst mean $\left|\Delta v_{\text {det }}\right| 10 \%$, orange: next $10 \%$, yellow: next $10 \%$ and gray: all the remaining values of the worst mean $\left|\Delta v_{\text {det }}\right|$. The blue points in the panel in row three on the left (the range-independent model underestimates the detection) are concentrated in the area of transition between the Scotian Shelf and deep water. Green and cyan points are located towards the deep water (between latitude of $41^{\circ} \mathrm{N}$ and $42^{\circ} \mathrm{N}$ ). The red, orange and yellow points are almost all located on the Scotian Shelf where the detection volume is most likely overestimated by the range-independent modeling. The largest outliers in the absolute terms (mean $\left|\Delta v_{\text {det }}\right|$ ) are located on the Scotian Shelf.

\section{Summation}

Returning to the original question, we can conclude that the choice of propagation modeltype should be carefully considered. A range-dependent model should be considered when interested in the performance of a single sonar at one very specific location, at a specific time, given a very tightly constrained set of environmental conditions, which include shallow water with significant variation in bathymetry. As shown in Fig. 6, the errors associated with range-dependent acoustic models can be significant, as well as the errors associated with assuming range independence in strongly varying environments. Generally speaking, for most acoustic studies considering the development of a sonar for general use across a variety of environments, the errors associated with using a range-independent acoustic model are too small to have profound impacts on study results. Realistically, large environmental variability can most often be captured in range-independent modeling. More specifically, this is the case for many studies considering the comparison of sonar systems — as long as a large number of environments are used. This conclusion is limited by the scope of this study and the data used, both in the geospatial sense, timeframe and the assumptions made.

In contrast, statistically speaking, many range predictions are very accurate when comparing range-dependent and range-independent environment representations. We have shown that in the range-independent case, if the required accuracy for detections is within $1 \mathrm{~km}$, over $60 \%$ of the results are within this error range, and $75 \%$ are within $10 \mathrm{~km}$. When the detection ranges are compared over a large number of conditions, the distributions of $r_{\text {det }}$ and $r_{\text {det0 }}$ are very similar (see Fig. 5). We have shown that a range-independent model can provide reasonable coverage across environmental variability, provided many environments are evaluated. Further, for a very specific environment, the range-independent model may provide results that are significantly incorrect, however, in an average sense, the results will be close to the more realistic varying results one would see in situ.

Similarly, a handful of environments may not lead to a representative comparison of two sonar systems because of the variability in the actual sound propagation conditions. For a given environment, one sonar may appear to have better detection ranges or higher 
probabilities of detection than the other by a significant amount. It is possible that this is not a robust result representative of an average performance difference. Conversely, lack of performance of one sensor in a specific environment may obscure the fact that the sensor is better on average across variable environments or possibly in the majority of conditions of interest. Due to the very large variability of sound propagation conditions, multiple environments should always be considered and the full distribution (not a single statistic or observation) of the measures of merit should be reported.

\section{Future Work}

The authors solely focused on comparing range-dependent with range-independent modeling assuming that the environment databases are accurate and that the ray-tracing and boundary scattering models are valid. If one should attempt to extrapolate from these findings, one must be cognizant of the fact that they will not be able to model exactly the future conditions based on the historical data used here. However, one could state the future predictions more in Bayesian fashion, whereby forecasts of detection performance can be evaluated sequentially, as environmental data become available to the researcher.

Monthly averages for the sea temperature and salinity (as functions of depth) were used for the month of September. The ESME database also contains the standard deviations of those values, which we did not use. The authors have interest in quantifying what the impact of those short-term SSP variations on the prediction of the detection ranges would be, however, this is likely a project that would require a significant time investment that is not currently an option.

Finally, the authors have some interest in developing this modeling effort to study the effects of ray bending across azimuths on the measures of merit considered here, time should become available. This is likely a considerable effort and should be thoroughly researched prior to taking on such a task.

\section{References}

1. F. B. Jenson, Numerical models of sound propagation in real oceans, in Proc. MTS/IEEE Oceans 82 Conf. (IEEE, New York, 1982), pp. 54-147.

2. M. Porter and H. Bucker, Gaussian beam tracing for computing ocean acoustic fields, J. Acoust. Soc. Am. 82(4) (1987) 1349-1359.

3. D. McCammon, User's guide to BellhopDRDC active v5 and updated passive v4a, DRDC Atlantic Contract Report (2011).

4. D. McCammon, User's guide to BellhopDRDC v4 active and passive versions, DRDC Atlantic Contract Report (2010).

5. M. Porter and Y. Liu, Finite-element ray tracing, in Proce. Int. Conf. Theoretical and Computational Acoustics (World Scientific, Singapore, 1994), pp. 947-956.

6. M. Porter, The Bellhop manual and user's guide, http://oalib.hlsresearch.com/Rays/HLS-20101.pdf. Accessed on June 2016.

7. O. C. Rodriguez, General description of the bellhop ray tracing program (2008), http://.hlsresearch.com/Rays/GeneralDescription.pdf. Accessed on February 2016. 
8. The Oceans Acoustics Library, Acoustic modeling software and data supported by the US Office of Naval Research, Ocean Acoustics program (2008), http://oalib.hlsresearch. com/Modes/AcousticsToolbox/at.zip. Accessed on January 2016.

9. P. Beckmann and A. Spizzichino, The Scattering of Electromagnetic Waves from Rough Surfaces (Pergamon Press, distributed in the Western Hemisphere by Macmillan, New York, 1963).

10. G. A. Liebiger, A combined ray theory normal mode approach to long range low frequency propagation loss prediction, Naval Underwater Systems Center, Technical Memo PA3-0109-71 (1971).

11. R. Urick, Principles of Underwater Sound (McGraw-Hill, New York, 1983).

12. Boston University Hearing Research Center and Office of Naval research Life Sciences Research Program, Effects of sound on the environment, digital bathymetry database (DBDB) v5.4 (2008), http://esme.bu.edu, Accessed on January 2016.

13. Boston University Hearing Research Center and Office of Naval research Life Sciences Research Program, Effects of sound on the environment, generalized digital environmental model (GDEM) v3.0, water temperature and water salinity database (2008), http://esme.bu.edu. Accessed on January 2016.

14. K. V. Mackenzie, Nine-term equation for the sound speed in the oceans, J. Acoust. Soc. Am. 70(3) (1981) 807-812.

15. R. Hodges, Underwater Acoustics: Analysis, Design and Performance of Sonar (Wiley, New York, 2010).

16. D. McCammon, An investigation of the Bellhop acoustic prediction model, capabilities, tests, recommendations and user guide, DRDC Atlantic Contract Report (2005). 